\title{
Undiagnosed Diabetes Mellitus and Associated Factors among Psychiatric Patients Receiving Antipsychotic Drugs at The University of Gondar Hospital, Northwest Ethiopia
}

\author{
Daniel Asmelash ${ }^{1 *}$, Wondale Getnet ${ }^{1}$, Belete Biadgo ${ }^{2}$, Sintayehu Ambachew ${ }^{2}$, \\ Tadele Melak ${ }^{2}$, Lemmesa Melese ${ }^{2}$, Shiwaneh Damite ${ }^{2}$, Habtamu Wondifraw \\ Baynes $^{2}$, Molla Abebe ${ }^{1}$
}

\section{OPEN ACCESS}

Citation: Daniel Asmelash, Wondale Getnet, Belete Biadgo, Sintayehu Ambachew, Tadele Melak, Lemmesa melese, Shiwaneh Damite, Habtamu Wondifraw Baynes, Molla Abebe. Undiagnosed Diabetes Mellitus and Associated Factors among Psychiatric Patients Receiving Antipsychotic Drugs at The University of Gondar Hospital, Northwest Ethiopia. Ethiop J Health Sci.2017;28(1):3.

doi:http://dx.doi.org/10.4314/ejhs.v28i1. 2

Received: June 15, 2017

Accepted: June 20, 2017

Published: January 1, 2018

Copyright: (C) 2018 Daniel Asmelash, et al. This is an open access article distributed under the terms of the Creative Commons Attribution License, which permits unrestricted use, distribution, and reproduction in any medium, provided the original author and source are credited.

Funding: University of Gondar Competing Interests: The authors declare that this manuscript was approved by all authors in its form and that no competing interest exists.

Affiliation and Correspondence:

${ }^{1}$ Department of Clinical Chemistry, School of Biomedical and Laboratory Sciences, College of Medicine and Health Sciences, University of Gondar, Gondar, Ethiopia

${ }^{2}$ University of Gondar Hospital Laboratory, College of Medicine and Health Sciences, University of Gondar, Gondar, Ethiopia

*Email: daniel.asmelash111@gmail.com

\section{ABSTRACT}

BACKGROUND: Undiagnosed diabetes mellitus cases are at higher risk for diabetic related complications. In low-income African countries, patients with undiagnosed diabetes mellitus account for $75 \%$ of diabetes cases. Psychiatric disorders have a greater impact on the global burden of diseases and disability associated with chronic diseases like diabetes mellitus and cardiovascular diseases.

METHODS: Institution based cross-sectional study was conducted at the University of Gondar Hospital from February to April 2016. $A$ total of 205 psychiatric patients aged above 15 years that were taking antipsychotic were included by the simple random sampling method. Fasting blood glucose, triglycerides and cholesterol level were determined from venous blood samples to evaluate diabetes mellitus based on WHO criteria.

RESULTS: Among 205 psychiatric patients taking antipsychotics, 15(7.3\%) had undiagnosed diabetes mellitus. Duration of antipsychotic treatment and sex had a statistically significant association with the prevalence of undiagnosed diabetes mellitus. As the duration of antipsychotic drug treatment increased by one year, the risk of having a diabetes mellitus increase by 1.47 times (AOR: 1.47 CI: 1.021-2.125).

CONCLUSION: The prevalence of undiagnosed diabetes mellitus among psychiatry patients taking antipsychotics was higher than the estimated diabetes national prevalence of Ethiopia. Screening of diabetes mellitus in particular, patients having a longer duration of antipsychotic treatment is mandatory to bring more undiagnosed cases for medical attention.

KEYWORDS: Diabetes mellitus, Psychiatric disorder, Antipsychotic 


\section{INTRODUCTION}

Diabetes mellitus (DM) is a complex metabolic disorder characterized by persistent hyperglycemia resulting from defects in insulin secretion, insulin action or both. There are four types of diabetes mellitus (DM), classified as type 1 diabetes mellitus, type 2 diabetes mellitus (T2DM), gestational diabetes and other types of $\mathrm{DM}(1)$.

In 2013, 382 million people had DM; this number is expected to rise to 592 million by 2035. Most people with the DM live in low and middle-income countries (2). Ethiopia ranked among the ten top countries in Africa with a higher prevalence of DM. According to International diabetic federation (IDF) estimated national diabetes prevalence was $4.36 \%$ in 2013 (3).

About $75 \%$ of the DM patients are estimated to be undiagnosed in low-income African countries (4). The undiagnosed DM cases, they remain untreated and they are one of the reasons for a poor glycemic control level of patients. This will cause diabetic complications, such as heart, kidney, and eye diseases, lower limb amputations and mental disorders $(3,5)$.

Psychiatric disorder or mental disorder is a clinically significant behavioral or psychological syndrome or pattern that occurs in an individual and associated with present distress or disability (6). In 2010, mental, neurological and substance use disorders accounted for 10.4\%, 2.3\% and, $28.5 \%$ of global disability life years, yet remain the lowest on the agenda of policy-makers, particularly in developing countries $(7,8)$. Study done in Ethiopia shows, the major psychiatric disorders are schizophrenia $(56.1 \%)$ followed by bipolar disorder $(20.6 \%)$ and major depressive disorder (MDD) (11.4\%) (8).

People with mental illness such as schizophrenia, depression or bipolar disorder have worse physical health and reduced life expectancy compared to the general population. This excess mortality is due to the fact that people with mental disorder are more likely to be overweight, and to have DM, hypertension and dyslipidemia $(9,10)$.
Despite the increased risk of DM and cardiovascular disease (CVD), many patients with mental disorder have limited access to general health care with less opportunity for CVD and DM risk screening and prevention than would be expected in non-psychiatric population $(11,12)$.

$\mathrm{DM}$ is highly prevalent in people with psychotic disorders, including schizophrenia and schizoaffective disorders. The exact prevalence is difficult to estimate since DM is often underdiagnosed in people with psychiatric disorders. The association between DM and psychosis is complex and multifactorial. Antipsychotic medication, poor lifestyle and genetic loading increase DM risk (12).

In addition, chronic stress is a risk factor in the incidence of DM and worsens metabolic control, which leads to diabetes-related complication. Patients with psychiatry disorder shows over-activation of both the hypothalamicpituitary-adrenal medullary axes. These endocrine systems respond to stress by an increase in the production of diabetogenic hormones cortisol and adrenaline. It is proposed that both hormones can raise blood sugar and play a part in insulin resistance and the onset of DM (13).

Research findings from different studies indicated that the prevalence of DM in psychiatric patients was higher than the one in the general population. Therefore, this study will provide information about the magnitude of undiagnosed DM in psychiatry patients taking antipsychotics since no sufficient studies have been done in Ethiopia.

\section{METHODS}

\section{Study area, study design, and study participants:}

An institution based cross-sectional study was carried out from February-April 2016 at the University of Gondar Hospital Psychiatry Clinic, which is found in Gondar Town. All psychiatric patients aged above 15 years, receiving antipsychotic drugs and voluntary to give informed consent were included. 
Sample size and sampling technique: A total of 205 individuals were enrolled in the study. Psychiatric patients aged $\geq 15$ years with a diagnosis of the major psychiatric problem were included. The simple random sampling technique was used to recruit study participants, who has been following antipsychotic treatment during the study period.

Data collection and laboratory methods: Data were collected by pretested and semi-structured Amharic version questionnaire using interview technique. During the data collection, patients who were taking antipsychotic drugs were interviewed by the enumerators using the questionnaire.

At every data collection spot, sufficient explanation about the aim of the research was given to the patients or caretakers of psychiatry patients. After informed consent was obtained, participants were interviewed face-to-face on the first visit, and blood samples were collected if they were in the fasting state. However, patients in the non-fasting state were instructed to be on overnight fasting eights up to ten hours. Then, three $\mathrm{ml}$ of blood sample was collected in a plain tube and centrifuged at 3000 RPM for 10 minutes, and serum was separated. The samples were analyzed within two hours using automated clinical chemistry analyzer (A25 BIOSYSTEM). In addition to the fasting plasma glucose (FPG), the laboratory testing included the levels of total blood cholesterol (TCHO) and triglycerides (TG). Based on the laboratory FBG result, diabetes mellitus was defined as FPG of $\geq$ $7.0 \mathrm{mmol} / 1$ (126mg/dl).

Quality assurance: Pre-test was done to check clarity, acceptability and consistency of the structured questionnaire. The reliability of the laboratory findings was assured by implementing quality control measures during the whole process of the laboratory works (preanalytical, analytical and post-analytical quality control steps were followed). All materials, equipment and procedures were adequately controlled. A control was run to check the functionality of clinical chemistry automation, which was used for conducting laboratory analysis.
Data processing and analysis: All patient data were registered in laboratory worksheet during the study period. Then, the data were cleaned, edited, checked for completeness and were entered to EPI info version 3.5.3 and transferred to the Statistical Package for Social Sciences (SPSS) statistical software version 20 for analysis.

The binary logistic regression model was used to examine the factors associated with undiagnosed DM among study participants. Variables with $p<0.2$ in the bivariate analysis were considered for multivariate analysis. Then, study findings were explained in words and tables. Finally, from a multivariate logistic regression model, variables with $\mathrm{P}$-value $<0.05$ were taken as statistically significant.

Ethical approval and consent to participate: Ethical clearance was obtained from the Ethical Review Board of the School of Biomedical and Laboratory Sciences, College of Medicine and Health Sciences, University of Gondar. Moreover, a letter of support was secured from administrators of the University of Gondar Hospital. Following an explanation of the purpose and the possible risks of the study, written consent was obtained from all study subjects, which assured that participation was on a voluntary basis. Participants with positive results for diabetes mellitus were referred for treatment to the Diabetic Clinic within the Hospital. The collected data were kept confidential.

\section{RESULTS}

Socio-demographic characteristics of the study participants: A total of 205 psychiatric patients taking antipsychotics were included in the study. Out of all the study participants, 108 $(52.7 \%)$ were females. The mean age of the study participants was $33.73 \pm 11.46$ (range: 15 to 81 years), and the majority $(60.5 \%)$ of the study participants were urban inhabitants (Table1).

of antipsychotic drug treatment was $2.62 \pm 1.8$ years. The mean duration of antipsychotic treatment for patients with an undiagnosed DM was Mean \pm SD of $4.4 \pm 1.53$ years. (Table 2). 
Table 1: Socio-demographic characteristics of psychiatric patients receiving antipsychotic drugs at the University of Gondar Hospital, northwest Ethiopia, 2016 ( $n=205)$.

\begin{tabular}{lll}
\hline Variable & Number & \% \\
\hline Sex & 97 & 47.3 \\
Male & 108 & 52.7 \\
Female & & \\
Age & 54 & 26.3 \\
$<25$ & 74 & 36.1 \\
$25-34$ & 42 & 20.5 \\
$35-45$ & 35 & 17.1 \\
$>45$ & & 52.2 \\
Marital Status & 107 & 33.1 \\
Single & 68 & 14.1 \\
Married & 29 & 0.5 \\
Separated/divorced & 1 & 49.3 \\
Widowed & & 19.0 \\
Educational Status & 101 & 18 \\
Illiterate & 39 & 13.7 \\
Read and write & 37 & \\
Primary \& secondary School & 28 & 83.4 \\
College and Above & & 14.6 \\
Religion & 171 & 2 \\
Orthodox & 30 & 39.5 \\
Muslim & 4 & 60.5 \\
Others & & \\
Residence & 81 & 124 \\
$\quad$ Rural & & \\
Urban & & \\
\hline
\end{tabular}

Behavioral characteristics of the study participants: The majority of the participants were schizophrenia patients, 106 (51.7\%), followed by MDD, 68 (33.2\%), and Bipolar disorder, 31 (15.1\%). Almost all of the study participants, 203 (99\%), had no family history of DM. Nine out of $15(60 \%)$ DM cases were schizophrenia patients, six out of $15(40 \%)$ undiagnosed DM cases were MDD patients and there was no case of undiagnosed DM in Bipolar patients. The mean duration of antipsychotic drug treatment was $2.62 \pm 1.8$ years. The mean duration of antipsychotic treatment for patients with undiagnosed DM was $4.4 \pm 1.53$ years. (Table 2).

Prevalence of undiagnosed diabetes mellitus: According to the ADA criteria, the prevalence of undiagnosed DM was $7.3 \%$ (CI: 3.74 - 10.86). Among participants with undiagnosed DM, the prevalence of undiagnosed DM was more prevalent in females than males $(5.9 \%$ vs. $1.5 \%)$ $(\mathrm{P}$-value $=0.028)$. The prevalence of undiagnosed DM increased across four age groups: $0 \%, 1.5 \%$, $2.4 \%$ and $3.4 \%$ among participants aged $<25,25$ $34,35-45$ and above 45 years respectively (Table $3)$. 
Table 2: Prevalence of undiagnosed diabetes mellitus by behavioral characteristics, clinical and anthropometric measurements of study participants at University of Gondar Hospital, northwest Ethiopia, 2016

\begin{tabular}{|c|c|c|c|c|}
\hline \multirow[t]{2}{*}{ Variable } & \multirow[t]{2}{*}{ Category } & \multirow{2}{*}{$\begin{array}{l}\text { Total } \\
\text { N (\%) }\end{array}$} & \multicolumn{2}{|c|}{ UDM } \\
\hline & & & $\begin{array}{c}\text { Yes } \\
\text { N }(\%)\end{array}$ & $\begin{array}{c}\text { No } \\
\text { N (\%) }\end{array}$ \\
\hline \multirow[t]{3}{*}{ Smoking status } & Non smoker & 191(93.2) & $14(6.8)$ & $177(86.3)$ \\
\hline & Current Smoker & $12(5.9)$ & $1(0.5)$ & $11(5.4)$ \\
\hline & Ex-smoker & $29(1)$ & $0(0)$ & $2(1)$ \\
\hline \multirow[t]{3}{*}{ Physical activity } & Light & $61(29.8)$ & $15(7.3)$ & $46(22.4)$ \\
\hline & Moderate & $105(51.2)$ & $0(0)$ & $105(51.2)$ \\
\hline & Vigorous & $39(19)$ & $0(0)$ & $39(19)$ \\
\hline \multirow[t]{3}{*}{ Blood Pressure } & Non hypertensive & $196(95.6)$ & $13(6.3)$ & $183(89.3)$ \\
\hline & Pre hypertensive & $7(3.4)$ & $1(0.5)$ & $6(2.9)$ \\
\hline & Hypertensive & $2(1)$ & $1(0.5)$ & $1(0.5)$ \\
\hline \multirow[t]{4}{*}{ BMI } & Underweight & $19(9.3)$ & $1(0.5)$ & $18(8.8)$ \\
\hline & Normal & $137(66.8)$ & $1(0.5)$ & $136(66.7)$ \\
\hline & Overweight & $38(18.5)$ & $11(5.4)$ & $27(13.2)$ \\
\hline & Obese & $10(4.9)$ & $2(1)$ & $8(3.9)$ \\
\hline \multirow[t]{2}{*}{ Family History of DM } & Yes & $2(1)$ & $0(0)$ & $2(1)$ \\
\hline & No & $203(99)$ & $15(7.3)$ & $188(91.7)$ \\
\hline \multirow[t]{3}{*}{ Type of disorder } & Schizophrenia & $106(51.7)$ & $9(4.4)$ & $97(47.3)$ \\
\hline & MDD with Psychotic & $68(33.2)$ & $6(2.9)$ & $62(30.2)$ \\
\hline & Bipolar with Psychotic & $31(15.1)$ & $0(0)$ & $31(15.1)$ \\
\hline \multirow[t]{4}{*}{ Antipsychotic used } & Typical & $92(44.9)$ & $8(3.9)$ & $84(41)$ \\
\hline & Atypical & $18(8.8)$ & $1(0.5)$ & $17(8.3)$ \\
\hline & Antipsychotic with Antidepressant & $69(33.7)$ & $6(2.9)$ & $63(30.7)$ \\
\hline & Antipsychotic with mood stabilizer & $26(12.7)$ & $0(0)$ & $26(12.7)$ \\
\hline \multirow[t]{2}{*}{ Blood TCHO } & Normal & $151(73.7)$ & $7(3.4)$ & $144(70.2)$ \\
\hline & High & $54(26.3)$ & $8(3.9)$ & $46(22.4)$ \\
\hline \multirow[t]{2}{*}{ Blood TG } & Normal & $167(81.5)$ & $9(4.4)$ & $158(77.1)$ \\
\hline & High & $38(18.5)$ & $6(2.9)$ & $32(15.6)$ \\
\hline
\end{tabular}

Factors associated with undiagnosed diabetes mellitus: In the bivariate analysis of variables with undiagnosed diabetes mellitus, sex, duration of antipsychotic treatment, total blood cholesterol and triglyceride were found to have a statistically significant association with the prevalence of undiagnosed DM.

However, in the multivariate logistic regression analysis of the study variables, there was a statistically significant association only with sex and duration of antipsychotic treatment. Females were found to have 7.1 times to be at risk of having undiagnosed DM than males (AOR: 7.1, CI: 1.4 - 36.1), and as the duration of antipsychotic drug treatment increased by one year, the risk of having DM increased by 1.47 units (AOR: 1.47, CI: 1.021-2.125) (Table 4). 
Table 3: Prevalence of undiagnosed diabetes mellitus by socio demographic characteristics of study participants at University of Gondar Hospital, northwest Ethiopia, 2016.

\begin{tabular}{lllc}
\hline Variable & Category & $\begin{array}{c}\text { UDM } \\
\text { Yes } \\
\text { Nex }\end{array}$ & $\begin{array}{c}\text { No }(\%) \\
\text { N(\%) }\end{array}$ \\
\hline \multirow{4}{*}{ Age } & Male & $3(1.5)$ & $94(45.9)$ \\
& Female & $12(5.9)$ & $96(46.8)$ \\
& $<25$ & $0(0.0)$ & $54(26.3)$ \\
Marital status & $25-34$ & $3(1.5)$ & $71(34.6)$ \\
& $35-45$ & $5(2.4)$ & $36(17.6)$ \\
& $>45$ & $7(3.4)$ & $29(14.1)$ \\
Religion & Married & $4(2)$ & $64(31.2)$ \\
& Single & $7(3.4)$ & $100(48.8)$ \\
Educational status & Divorced/separated & $4(2)$ & $25(12.2)$ \\
& Widowed & $0(0)$ & $1(0.5)$ \\
& Orthodox & $12(5.9)$ & $159(77.6)$ \\
Residence & Muslim & $2(1)$ & $28(13.7)$ \\
& Others & $1(0.5)$ & $3(1.5)$ \\
& Illiterate & $11(5.4)$ & $90(43.9)$ \\
& Read and write & $0(0.0)$ & $39(19)$ \\
& $1^{0}$ and $2^{0}$ school & $3(1.5)$ & $34(16.5)$ \\
& College and above & $1(0.5)$ & $27(13.2)$ \\
& Rural & $4(2)$ & $77(37.6)$ \\
& Urban & $11(5.4)$ & $113(55.1)$ \\
\hline
\end{tabular}

Table 4: Multivariate and bivariate analysis of factors associated with undiagnosed diabetes mellitus among psychiatry patients taking antipsychotic drugs at the University of Gondar Hospital, northwest Ethiopia, 2016

\begin{tabular}{lllllll}
\hline Variable & Category & \multicolumn{2}{c}{ Undiagnosed DM } & COR (95\% CI) & AOR (95\% CI) & P-value \\
& & Yes & No & & & \\
& & N (\%) & N (\%) & & & \\
Sex & Male & $3(1.5)$ & $94(45.9)$ & 1 & & \\
& Female & $12(5.9)$ & $96(46.8)$ & $3.91(1.07-14.32)^{*}$ & $7.1(1.436-35.4)$ & $0.016^{* *}$ \\
BMI & Under & $1(0.5)$ & $18(8.8)$ & 1 & & 0.002 \\
& Normal & $1(0.5)$ & $136(66)$. & $0.132(0.008-2.2)$ & $0.079(0.004-1.49)$ & 0.091 \\
& Overweight & $11(5.4)$ & $27(13.2)$ & $7.33(0.87-61.84)$ & $7.1(0.68-74.51)$ & 0.102 \\
\multirow{2}{*}{ Duration } & Obese & $2(1)$ & $8(3.9)$ & $4,5(0.355-57.10)$ & $2.18(0.133-36.06)$ & 0.585 \\
TCHO & Years of drug use & - & - & $1.44(1.157-1.80)^{*}$ & $1.47(1.021-2.12)^{* *}$ & $0.038^{* *}$ \\
& Normal & $7(3.4)$ & $144(70)$ & 1 & & \\
TG & High & $8(3.9)$ & $46(22.4)$ & $3.57(1.23-10.40)^{*}$ & $0.22(0.036-1.408)$ & 0.111 \\
& Normal & $9(3.4)$ & $158(77)$ & 1 & & \\
\hline
\end{tabular}

NB: $*=$ Significant by bivariate analysis, $* *=$ significant by multivariate analysis 


\section{DISCUSSION}

In this study, of 205 psychiatric patients, 15 (7.3\%) met the WHO criteria for the DM. The prevalence of undiagnosed DM (7.3\%) found in this study was higher than the estimated DM national prevalence of Ethiopia, $4.36 \%$ reported by the IDF in 2013 (3). This may be associated with poor health habits, low access to health care, the diabetogenic effect of the antipsychotic drugs and the global increase in the trend of the DM epidemic in developing countries.

The prevalence of undiagnosed DM among patients with psychiatric disorder in this study, (7.3\%) (CI: 3.74 - 10.86), was lower than the findings of the studies among psychiatric patients in Palestine (10.8\%) (14), Australia (12.1\%) (15) and USA (10.2\%) (16). It is likely that the differences observed in these studies were having a higher national prevalence of DM which could be due to lifestyle and genetic factors.

To the contrary, the prevalence $(7.3 \%)$ of undiagnosed DM was also higher than the study finding in mental health care organizations in Holland, $6.5 \%$ (17), University of Rochester Medical Center London, $4.9 \%$ (18) and in Srilanka, $4.75 \%$ (19). This could be due to deficiency in DM screening and early diagnosis because of insufficient resources and practical considerations.

A higher prevalence of undiagnosed DM was observed in females $(5.9 \%)$ than in males $(1.5 \%)$, which is consistent with a study carried out on psychiatric patients in China (Male $=18.3 \%$ vs. Female $=31.3 \%)(20)$ and USA (Males $=9.5 \%$ vs. Females $=14.7 \%)(21)$. The possible reason for a highly represented undiagnosed DM among female psychiatric patients in this study was due to poor health seeking behavior and adopting a sedentary lifestyle.

Undiagnosed DM was significantly associated with duration of antipsychotic treatment in which as the duration of antipsychotic drug treatment increased by one year, the risk of having undiagnosed DM increased by1.47 (AOR: 1.47, CI: 1.021-2.125). The finding of this study was also supported by a study done in South London, which showed an association of duration of antipsychotic treatment with an increased risk of DM (OR 1.01) (22). The hypothesized diabetogenic effects of longer duration of antipsychotic treatment were on antipsychotic-induced weight gain. This starts with the eventual development of insulin resistance and pancreatic beta-cell failure (23).

Limitation of the study: Due to financial constraints, we were unable to incorporate tests like oral glucose tolerance test (OGTT). OGTT can increase the specificity of the study besides only the FPG test was used to evaluate undiagnosed DM this could underestimate the prevalence of undiagnosed DM. In addition, we used cross-sectional study design and this may not show exactly the cause and effect relationship between variables.

In conclusion, this study has found a higher prevalence of undiagnosed DM among psychiatry patients taking antipsychotics than the one in the general population of Ethiopia according to IDF 2014 estimated report. The risk factors associated with the prevalence of undiagnosed DM were the duration of antipsychotic drug treatment and sex of the participants.

We reccomend early diagnosis and screening of DM in psychiatric patients, in particular, those patients with advanced age and longer duration of antipsychotic treatment. This will have a major importance to reduce the risk of DM-related complications like cardiovascular diseases by adopting lifestyle intervention.

\section{ACKNOWLEDGEMENTS}

We would like to thank the study participants for voluntarily participating in the study. We are also grateful to the University of Gondar Hospital for allowing us to use the laboratory facilities.

\section{REFERENCES}

1. Association AD. Diagnosis and classification of diabetes mellitus. Diabetes care. 2014;37(Supplement 1):S81-S90.

2. Guariguata L, Whiting D, Hambleton I, Beagley J, Linnenkamp U, Shaw J. Global estimates of diabetes prevalence for 2013 and projections for 2035. Diabetes research and clinical practice. 2014;103(2):137-49.

3. Aguiree F, Brown A, Cho NH, Dahlquist G, Dodd S, Dunning T, et al. IDF diabetes atlas. 2013.

4. Chiwanga FS, Njelekela MA, Diamond MB, Bajunirwe F, Guwatudde D, Nankya-Mutyoba J, 
et al. Urban and rural prevalence of diabetes and pre-diabetes and risk factors associated with diabetes in Tanzania and Uganda. Global health action. 2016;9(1):31440.

5. Buowari OY. Diabetes mellitus in developing countries and case series. Diabetes MellitusInsights and Perspectives: InTech; 2013.

6. Stein DJ, Phillips KA, Bolton D, Fulford K, Sadler $\mathrm{JZ}$, Kendler KS. What is a mental/psychiatric disorder? From DSM-IV to DSM-V. Psychological medicine. 2010;40(11):1759-65.

7. Whiteford HA, Ferrari AJ, Degenhardt L, Feigin $\mathrm{V}$, Vos T. The global burden of mental, neurological and substance use disorders: an analysis from the Global Burden of Disease Study 2010. PloS one. 2015;10(2): 0116820.

8. Fekadu A, Desta M, Alem A, Prince M. A descriptive analysis of admissions to Amanuel Psychiatric Hospital in Ethiopia. Ethiopian Journal of Health Development. 2007;21(2):173-8.

9. Nasrallah HA, Meyer JM, Goff DC, McEvoy JP, Davis SM, Stroup TS, et al. Low rates of treatment for hypertension, dyslipidemia and diabetes in schizophrenia: data from the CATIE schizophrenia trial sample at baseline. Schizophrenia research. 2006;86(1):15-22.

10. Correll CU, Detraux J, De Lepeleire J, De Hert M. Effects of antipsychotics, antidepressants and mood stabilizers on risk for physical diseases in people with schizophrenia, depression and bipolar disorder. World Psychiatry. 2015;14(2):119.

11. De Hert M, Dekker J, Wood D, Kahl K, Holt R, Möller H-J. Cardiovascular disease and diabetes in people with severe mental illness position statement from the European Psychiatric Association (EPA), supported by the European Association for the Study of Diabetes (EASD) and the European Society of Cardiology (ESC). European psychiatry. 2009;24(6):412-24.

12. Ward M, Druss B. The epidemiology of diabetes in psychotic disorders. The Lancet Psychiatry. 2015;2(5):431-51.

13. Bădescu S, Tătaru C, Kobylinska L, Georgescu E, Zahiu D, Zăgrean A, et al. The association between Diabetes mellitus and Depression. Journal of medicine and life. 2016;9(2):120.

14. Sweileh WM, Dalal SA, Sa'ed HZ, Al-Jabi SW, Al-Ali I. Diabetes mellitus in patients with schizophrenia in West-Bank, Palestine. diabetes research and clinical practice. 2013;99(3):351-7.

15. Foley DL, Mackinnon A, Morgan VA, Watts GF, McGrath JJ, Castle DJ, et al. Predictors of type 2 diabetes in a nationally representative sample of adults with psychosis. World Psychiatry. 2014;13(2):176.

16. Manu P, Correll CU, van Winkel R, Wampers M, De Hert M. Prediabetes in patients treated with antipsychotic drugs. Journal of Clinical Psychiatry. 2012;73(4):460.

17. Cohen D, Stolk RP, Grobbee DE, Gispen-de Wied CC. Hyperglycemia and diabetes in patients with schizophrenia or schizoaffective disorders. Diabetes care. 2006;29(4):786-91.

18. Lamberti JS, Costea GO, Olson D, Crilly JF, Maharaj K, Tu X, et al. Diabetes mellitus among outpatients receiving clozapine: prevalence and clinical-demographic correlates. Journal of Clinical Psychiatry. 2005;66(7):900-6.

19. Hanwella R, de Silva V. Diabetes and impaired fasting glucose in Sri Lankan patients with schizophrenia. Sri Lanka Journal of Psychiatry. 2010;1(2).

20. Zhang R, Hao W, Pan M, Wang C, Zhang X, Chen $\mathrm{DC}$, et al. The prevalence and clinical-demographic correlates of diabetes mellitus in chronic schizophrenic patients receiving clozapine. Human Psychopharmacology: Clinical and Experimental. 2011;26(6):392-6.

21. Citrome L, Jaffe A, Levine J, Allingham B, Robinson J. Relationship between antipsychotic medication treatment and new cases of diabetes among psychiatric inpatients. Psychiatric Services. 2004;55(9):1006-13.

22. Taylor D, Young C, Mohamed R, Paton C, Walwyn R. Undiagnosed impaired fasting glucose and diabetes mellitus amongst inpatients receiving antipsychotic drugs. Journal of Psychopharmacology. 2005;19(2):182-6.

23. Scigliano G, Ronchetti G. Antipsychotic-induced metabolic and cardiovascular side effects in schizophrenia: a novel mechanistic hypothesis. CNS drugs. 2013;27(4):249-57.

DOI: http://dx.doi.org/10.4314/ejhs.v28i1.2 